\title{
Antibacterial antibody responses associated with the development of asthma in house dust mite-sensitised and non-sensitised children
}

\author{
Belinda J Hales, ${ }^{1}$ Lee Ying Chai, ${ }^{1}$ Claire E Elliot, ${ }^{1}$ Leigh J Pearce, ${ }^{1}$ Guicheng Zhang, \\ Tatjana K Heinrich, ${ }^{1}$ Wendy-Anne Smith, ${ }^{1}$ Merci M Kusel, ${ }^{1}$ Patrick G Holt, ${ }^{1}$ \\ Peter D Sly, ${ }^{2}$ Wayne R Thomas ${ }^{1}$
}

\begin{abstract}
- Additional data are published online only. To view these files please visit the journal online (http://thorax.bmj.com/content/ 67/4.toc)

${ }^{1}$ Telethon Institute for Child Health Research and Centre for Child Health Research,

University of Western Australia, Subiaco, Western Australia,

Australia

${ }^{2}$ Queensland Children's Medical Research Institute, The University of Queensland, Royal Children's Hospital, Herston, Queensland, Australia
\end{abstract}

Correspondence to Dr B J Hales, Telethon Institute for Child Health Research and Centre for Child Health Research, University of Western Australia, 100 Roberts Road, Subiaco, WA 6008, Australia; belinda@ichr.uwa.edu.au

Received 26 June 2011 Accepted 28 October 2011 Published Online First 21 November 2011

\section{ABSTRACT}

Background Infants who develop house dust mite (HDM) allergy and HDM-sensitised children with severe persistent asthma have low antibody responses to the P6 antigen of Haemophilus influenzae.

Objective To measure the development of antibody to two ubiquitous bacteria of the respiratory mucosa in a prospective birth cohort at high risk of allergic disease and to assess which responses are associated with asthma and atopy.

Methods lgG1 and lgG4 antibody to $H$ influenzae (P4 and P6) and Streptoccocus pneumoniae (PspA and PspC) surface antigens was measured in yearly blood samples of children aged $1-5$ years. IgE to the P6 antigen was examined for the 5 -year group. The children were stratified based on HDM sensitisation and asthma at 5 years of age.

Results HDM-sensitised children had lower IgG1 antibody titres to the bacterial antigens, and early responses $(<3$ years and before the development of HDM sensitisation and asthma) corrected for multiple antigens were significantly reduced for $\mathrm{P} 4, \mathrm{P} 6$ and $\mathrm{PspC}$ $(p=0.008, p=0.004$ and $p=0.028$, respectively). Similar associations with asthma were also found $(p=0.008$, $p=0.004$ and $p=0.032$ for $\mathrm{P} 4, \mathrm{P} 6$ and $\mathrm{PspC}$, respectively). The IgG4 antibody titre and prevalence were similar in both HDM-sensitised and non-sensitised groups, but sensitised children had a slower downregulation of the IgG4 response. Children with asthma (27/145 at 5 years) had lower anti-P6 IgE responses $(p<0.05)$

Conclusions HDM-sensitised children have early defective antibody responses to bacteria that are associated with asthma. Surprisingly, antibacterial lgE was associated with a reduced risk for asthma.

\section{INTRODUCTION}

There is a strong association between allergy to indoor allergens and the development of asthma, but as many as $50 \%$ of children with high IgE antibodies do not develop disease. ${ }^{1}$ It is becoming apparent that the development of allergy and asthma is associated with deviated immune responses to respiratory viruses ${ }^{2} 3$ and mucosal bacteria ${ }^{4}$ which, through several proposed mechanisms, could increase susceptibility to sensitisation and disease. ${ }^{67}$ In keeping with this, children

\section{Key messages}

What is the key question?

- Do children who develop asthma and house dust mite sensitisation have altered immune responses to colonising bacteria early in life?

What is the bottom line?

- Early impaired antibacterial $\lg \mathrm{G} 1$ antibody is associated with atopy and asthma. The development of antibacterial $\lg E$ antibody is associated with protection from asthma.

Why read on?

- Altered immune responses in the development of asthma are not just restricted to viruses.

with atopic asthma experience more frequent and severe rhinovirus-induced illnesses ${ }^{8}$ and children who develop wheeze have increased bacterial colonisation of the nasopharynx as neonates. ${ }^{9}$ Additionally, the airways of people with asthma, once thought to be sterile, carry a larger load of Haemophilus influenzae than those of healthy people. ${ }^{10}$ The high prevalence of rhinovirus during asthma exacerbations ${ }^{8}$ and increased asthma found in children who develop wheezy rhinovirus infections early in life ${ }^{11}$ has provided a platform for ongoing investigations. The study of bacteria has not been as extensive, but colonisation with $H$ influenzae has been found to precede wheezing and also to be associated with exacerbations and to be independent of viral infection. ${ }^{12}$

In keeping with these associations, IgG1 antibody responses to the protective P6 antigen of $H$ influenzae have been found to be decreased in 2 -year-old children who developed atopy at 5 years. ${ }^{4}$ House dust mite (HDM)-sensitised children with frequent or persistent asthma exacerbations were also shown to have lower anti-P6 IgG1 titres than children with infrequent episodic asthma. ${ }^{5}$ One-third of atopic children and adults had the Th2-dependent IgG4 anti-P6 antibody. ${ }^{4}$ Antibacterial IgE antibody has also been found in both atopic and non-atopic subjects ${ }^{4} 13$ and, counterintuitively, for atopic subjects was inversely related to the risk of asthma. ${ }^{13}$ 
This paper describes the development of the antibody responses to the $\mathrm{P} 4$ and $\mathrm{P} 6$ antigens of $H$ influenzae and pneumococcal surface proteins A and C (PspA and PspC) of Streptococcus pneumoniae in a high-risk cohort of children. The primary aim of the study was to determine if the IgG antibody responses that we had noted previously in our studies with P6 ${ }^{4}$ occurred for different antigens and for different bacteria, and to observe when they occurred. The second aim was to determine if the inverse association of $\operatorname{IgE}$ antibody and asthma found in another cohort $^{13}$ also occurred in this cohort and if it was present early (5 years)

\section{METHODS}

\section{Characteristics of the study population}

Antibodies were examined in the plasma of children from a prospective birth cohort (Childhood Asthma Study) at 1, 2, 3, 4 and 5 years of age. A total of 263 children at high risk of atopy (at least one parent with a doctor-diagnosed history of hay fever, asthma or eczema) were recruited prenatally between 1996 and 1999. ${ }^{14}$ Skin prick tests (SPT) to common allergens were performed. A third of this population was HDM-sensitive ${ }^{14}$ with few $(<5 \%)$ being SPT-positive only to other allergens. The SPT-positive infants were selected solely on the presence of a positive skin test to HDM extract $(\geq 2.5 \mathrm{~mm})$ and the SPTnegative infants were selected on the absence of a skin test response to HDM at 5 years of age (table 1). Children without SPT information at 5 years were not included in the present study, and plasma and SPT data were available for 191, 188, 187, 176 and 145 children, respectively, from years 1 to 5 . The selected children were representative of the whole cohort based on variables such as HDM sensitisation, current asthma and gender. Asthma was defined as current doctor-diagnosed asthma and wheeze in the 12 months before the 3-, 4- or 5-year visit. Symptomatic infections, which were mostly viral, were recorded and reported by Kusel et $a l^{15}$ and are summarised in the online supplement. Samples were not collected for bacterial (and viral) carriage.

\section{Antigens}

The bacterial antigens were conserved surface proteins known to elicit protective immune responses that should have similar requirements for antigen processing and $\mathrm{T}$ cell responses as the allergens. Details of the antigen preparations for P4 and P6 from $H$ influenzae, PspA1, PspA2 and PspC from $S$ pneumoniae and the major HDM allergens are outlined in the online supplement.

\section{Quantification of antibody binding}

The IgE, IgG1 and IgG4 antibody isotypes to the bacterial antigens and IgE binding to the major HDM allergens Der $p 1$ and Der p 2 were assayed by a procedure using humanised chimeric antibodies for absolute quantitation. ${ }^{16} 17$ A microtitre plate dissociation-enhanced immunofluorescence assay (DELFIA) was performed where antigen coating was standardised by capturing His-tagged recombinant antigens with antiHis monoclonal antibody. The assay was calibrated by interpolating the results from a titration curve constructed with recombinant Der p 2 captured by the same procedure and a standardised (IU/ml) humanised chimeric IgE, IgG1 or IgG4 anti-Der p 2 antibody (Indoor Biotechnologies, Charlottesville, Virginia, USA). Antibody values below the limits of detection were ascribed a value of half the limit of detection.

Total IgE was quantitated by ImmunoCAP (Phadia, Uppsala, Sweden) in the Immunology Department at Princess Margaret Hospital. IgG1 was quantitated by DELFIA using antibody pairs from BD Biosciences (San Jose, California, USA) and an IgG1 plasma standard curve ranging from 0.02 to $1.64 \mathrm{mg} / \mathrm{ml}$.

\section{Statistical analysis}

Differences in the level of antibody binding by selected groups (SPT+ /SPT - or asthmatic/non-asthmatic at 5 years) were initially compared by Mann-Whitney or $\chi^{2}$ tests. Correlations were studied using the Spearman rank correlation. In order to investigate the association of asthma and HDM sensitisation at age 5 with the levels of antibody binding in the first 3 years of life and the first 5 years of life, further analysis with generalised estimating equations (GEE) were employed. The analyses accounted for inherent covariance in the same subject. In the GEE regression analyses, levels of antibody binding were logtransformed to approximate a normal distribution. All analyses were performed using GraphPad Prism Software (La Jolla, California, USA) and StataIC V.11 (StataCorp).

\section{RESULTS}

\section{IgG1 antibody development}

IgG1 antibody titres to P4 and P6 of $H$ influenzae and PspA (PspA1 and PspA2) and PspC of $S$ pneumoniae were measured in samples taken from 1 to 5 years (figure 1, table 2). Atopic children (SPT-positive to HDM at 5 years) showed decreased titres to P4 early in development at years 1 and 2. The mean P6 titres of the atopic group were about half of the non-atopic group for all years. The prevalence in the cohort (figure $2 \mathrm{~A}$ ) shows that responses were slow to develop with only $70 \%$ showing detectable titres at year 4 . The IgG1 titres of atopic children to the $S$ pneumoniae antigens showed reduced responses from years 2 to 3 for PspA (49\% of SPT-negative) and from years 1 to 2 for PspC (34\% of SPT-negative). There was a significantly reduced prevalence of antibodies at year 2 for PspC but not PspA. PspC induced high titres earlier than the other antigens and reached 10-20-fold higher titres than P4 and PspA at year 5, and these in turn were approximately 10 -fold higher than the titres to P6.

Table 1 Characteristics of the study population

\begin{tabular}{|c|c|c|c|c|c|}
\hline & 1 year old & 2 years old & 3 years old & 4 years old & 5 years old \\
\hline \multicolumn{6}{|c|}{ Boy/girls, n (\%) } \\
\hline SPT+ & $44 / 20(68 / 32)$ & $44 / 19(70 / 30)$ & $43 / 19(69 / 31)$ & $41 / 19(68 / 32)$ & $33 / 16(66 / 34)$ \\
\hline SPT- & $62 / 65(49 / 51)^{* *}$ & $62 / 63(50 / 50)^{* *}$ & $62 / 63(50 / 50)^{*}$ & $60 / 59(50 / 50)^{*}$ & $46 / 50(48 / 52)^{*}$ \\
\hline \multicolumn{6}{|c|}{ Asthma, n (\%) } \\
\hline SPT+ & ND & ND & $5 / 62(8.1)$ & $12 / 60(20.0)$ & $14 / 49(28.6)$ \\
\hline SPT- & ND & ND & $13 / 125(10.4)$ & $11 / 119\left(9.2^{*}\right)$ & $13 / 96\left(13.5^{*}\right)$ \\
\hline
\end{tabular}

The values in parentheses are the percentage of boys/girls or the percentage of children with asthma

Details of when asthma first started for individual subjects is outlined in the supplementary methods.

${ }^{*} p<0.05,{ }^{* *} p<0.001$ between SPT-positive and SPT-negative groups.

ND, not determined; SPT, skin prick test. 
Figure 1 Development of $\lg \mathrm{G} 1$ antibody (ng/ml) to the P4 and P6 antigens of Haemophilus influenzae and the PspA and PspC antigens of Streptococcus pneumoniae in plasma collected from children aged $1-5$ years. The mean antibody titre of SPT-positive (filled circles) and SPT-negative (open circles) children is shown. ${ }^{*} p<0.05$, ${ }^{* *} \mathrm{p}<0.01$, ${ }^{* *} \mathrm{p}<0.001$ between-group comparisons (SPT-positive vs SPTnegative). Lower $\lg \mathrm{G} 1$ titres to $\mathrm{P} 6$ at 1 , 3 and 5 years and PspC at 2 years were significant after correcting for multiple testing. The geometric mean and 95\% Cls are shown in table 2. SPT, skin prick test.
P4 $H$ influenzae

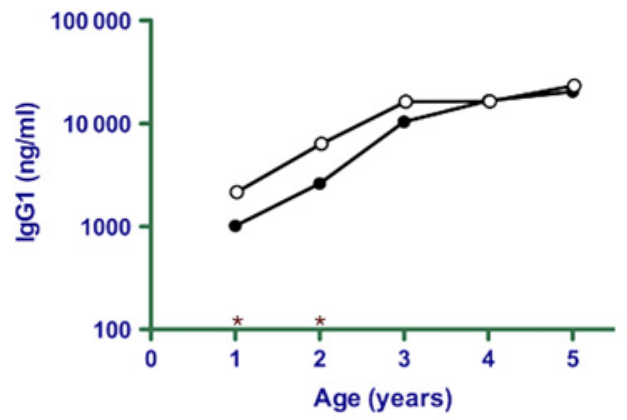

PspA (1\&2) S pneumoniae

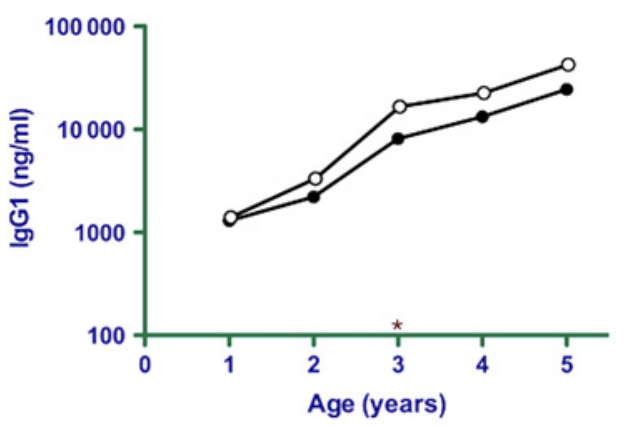

P6 $H$ influenzae

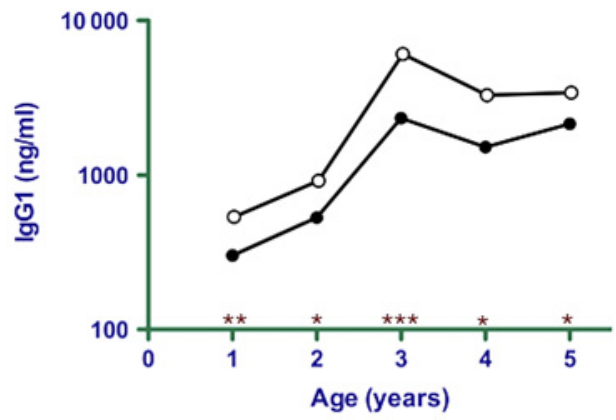

PspC S pneumoniae

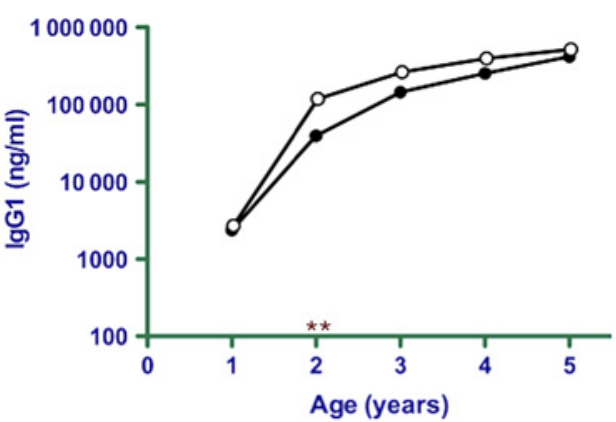

$\mathrm{SPT}+\infty \mathrm{SPT}-$

When the children were stratified for asthma at 5 years, the group with asthma had lower anti-P6 IgG1 titres from age 2 years $(p<0.05$ to $p=0.005)$ and lower anti-P4 at age 5 years $(p<0.01$, table 3$)$. The mean anti-PspC IgG1 titres at 2 years were 2.5 -fold lower in the asthma group, and a similar trend was observed for the anti-PspA titres between the ages of 2 and 4 years and for anti-P4 at age 4 years.

Since the early time points were shown to be important in the initial analyses, we further analysed the associations of HDM sensitisation and asthma at age 5 years with IgG1 antibody responses in the first 3 years of life using GEE regression approaches. The early time points in the first 3 years of life were also before the majority of children developed asthma. Low IgG1 titres to P4, P6 and PspC in the first 3 years of life were significantly associated with HDM sensitisation at age 5 , with a significance of $p=0.008, p=0.004$ and $p=0.028$, respectively, after correcting for multiple testing. Likewise, low anti-P4, anti-P6 and anti-PspC IgG1 antibody in the first 3 years of life were significantly associated with asthma at age 5 , with a significance of $p=0.008, p=0.004$ and $p=0.032$, respectively. Similar associations with HDM sensitisation and asthma and low IgG1 antibody responses to P4, P6 and PspC were observed in the first 5 years of life.

Overall, the IgG1 titres to P4 and P6 in different individuals were highly and very significantly correlated $(p<0.001)$, as were the titres to the PspA and PspC antigens $(p<0.005$; see table 1 in online supplement). However, the titres to the antigens of the

Table 2 IgG1 antibody titre stratified by HDM sensitisation (SPT-positive or SPT-negative at 5 years of age)

\begin{tabular}{|c|c|c|c|c|c|}
\hline & 1 year old & 2 years old & 3 years old & 4 years old & 5 years old \\
\hline \multicolumn{6}{|l|}{ P4 } \\
\hline SPT+ & 1022 (666 to 1522$)$ & 2617 (1494 to 4583 ) & 10458 (6676 to 16383 ) & 16698 (8532 to 32681 ) & 20447 (12932 to 32330 ) \\
\hline SPT- & 2168 (1483 to 3168$)$ & $6393(4044$ to 10106$)$ & 16391 (12020 to 22353$)$ & 16423 (10 123 to 26643$)$ & 23354 (16594 to 32866 ) \\
\hline $\mathrm{p}$ Value & 0.0406 & 0.0353 & 0.1010 & 0.9597 & 0.4891 \\
\hline \multicolumn{6}{|l|}{ P6 } \\
\hline SPT + & 303 (256 to 358 ) & 531 (376 to 750) & 2337 (1549 to 3525$)$ & 1521 (977 to 2368) & 2151 (1399 to 3309) \\
\hline SPT- & 538 (425 to 683 ) & 922 (682 to 1246$)$ & 6086 (4499 to 8233 ) & 3297 (2295 to 4737 ) & 3419 (2515 to 4648$)$ \\
\hline p Value & 0.0056 & 0.0434 & $<0.001$ & 0.0122 & 0.0487 \\
\hline \multicolumn{6}{|c|}{ PspA (1 and 2) } \\
\hline SPT+ & 1310 (997 to 1720$)$ & 2222 (1437 to 3437$)$ & 8144 (4902 to 13530$)$ & 13317 (6724 to 26373$)$ & 24524 (11799 to 50974$)$ \\
\hline SPT- & 1406 (1141 to 1733$)$ & 3347 (2308 to 4854$)$ & 16657 (11297 to 24560$)$ & 22581 (13473 to 37846$)$ & $42513(26510$ to 68174$)$ \\
\hline $\mathrm{p}$ Value & 0.7825 & 0.2986 & 0.0234 & 0.2498 & 0.1971 \\
\hline \multicolumn{6}{|l|}{ PspC } \\
\hline SPT+ & 2391 (1632 to 3504) & 39702 (20983 to 75121$)$ & 144959 (76 288 to 275444$)$ & 254361 (151998 to 425663 ) & $419481(250989$ to 701081$)$ \\
\hline SPT- & 2719 (2040 to 3625$)$ & 118443 (81380 to 172387$)$ & 263382 (182 130 to 380882 ) & 396132 (280887 to 558661 ) & 516873 (402468 to 663797$)$ \\
\hline $\mathrm{p}$ Value & 0.6685 & 0.0042 & 0.1644 & 0.1463 & 0.8886 \\
\hline
\end{tabular}

The value is the geometric mean of the $\operatorname{lgG} 1$ antibody titre $(\mathrm{ng} / \mathrm{ml})$ with the $\mathrm{Cl}$ of antibody binding in parentheses.

Comparisons between SPT-positive and SPT-negative groups by the Mann-Whitney test are shown.

p Values $<0.0125$ are considered significant after correcting for multiple testing.

HDM, house dust mite; SPT, skin prick test. 
Figure 2 Prevalence (\%) of (A) $\lg \mathrm{G} 1$ and (B) IgG4 antibody binding to antigens of Haemophilus influenzae and Streptococcus pneumoniae in plasma collected from SPT-positive (filled circles) and SPT-negative (open circles) children aged $1-5$ years. ${ }^{*} \mathrm{p}<0.05$,

${ }^{* *} \mathrm{p}<0.001$ between-group comparisons (SPT-positive vs SPT-negative).
A

P4 $\mathrm{H}$ influenzae

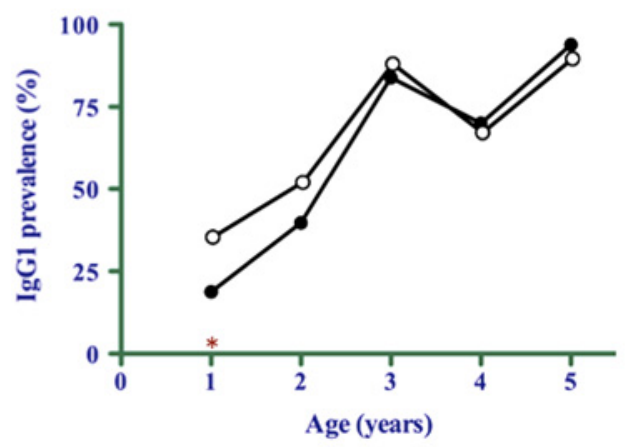

PspA (1\&2) S pneumoniae

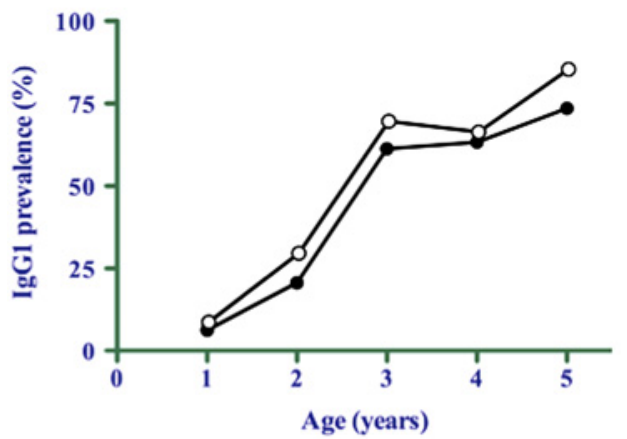

B

P4 $\mathrm{H}$ influenzae

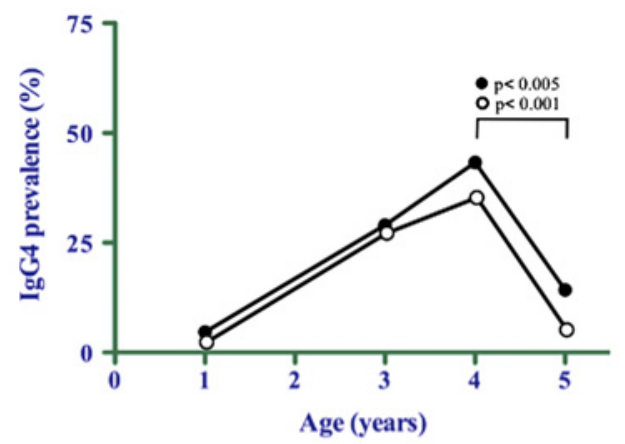

PspA (1\&2) S pneumoniae

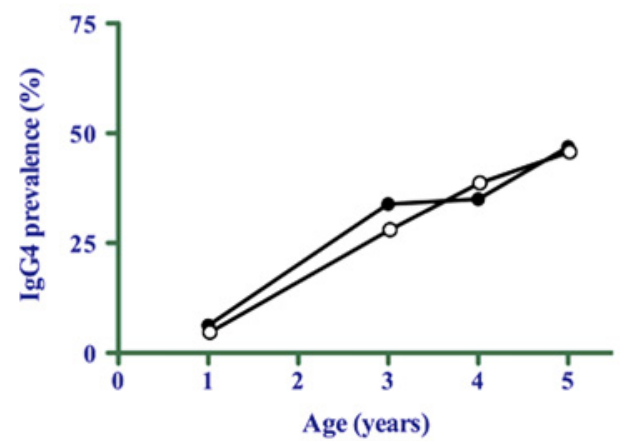

P6 $\mathrm{H}$ influenzae

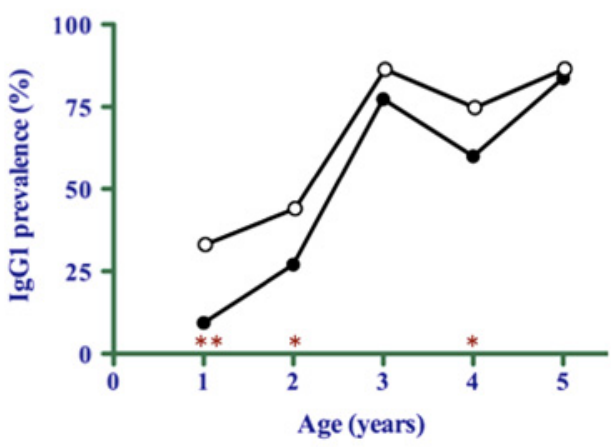

PspC S pneumoniae

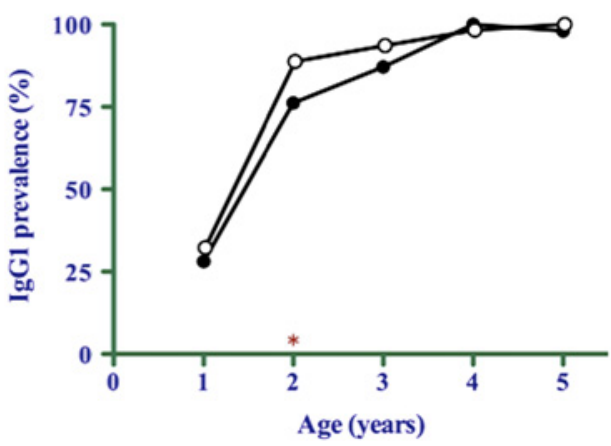

P6 $\mathrm{H}$ influenzae

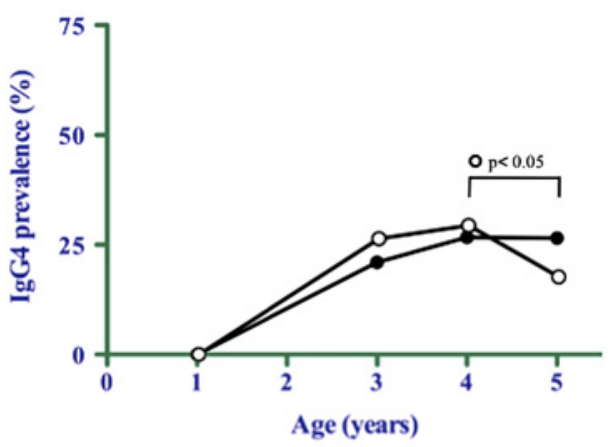

PspC S pneumoniae

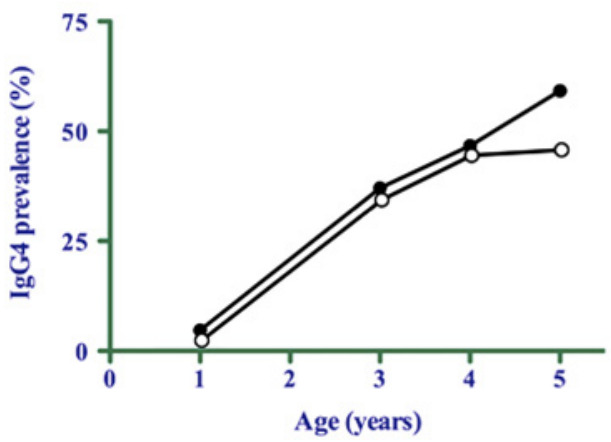

different bacteria were not well correlated, although some time points were significant $(\mathrm{p}<0.05)$.

\section{IgG4 development}

The titres of IgG4 antibody were low for most subjects (see table 2 in online supplement) and there were many non- responders, so the analysis has been presented as prevalence (figure 2B). No differences between the IgG4 responses of HDM-sensitised and non-sensitised subjects were found for P4, P6, PspA or PspC. There was, however, a gender difference for the response to P6 at 5 years of age with 20/79 boys and $8 / 66$ girls having detectable titres $(p<0.05)$. The 
Table $3 \quad \lg \mathrm{G} 1$ antibody titre stratified by asthma at 5 years of age

\begin{tabular}{|c|c|c|c|c|c|}
\hline & 1 year old & 2 years old & 3 years old & 4 years old & 5 years old \\
\hline \multicolumn{6}{|l|}{ P4 } \\
\hline Asthma & 1241 (605 to 2543 ) & 2771 (1133 to 6777$)$ & 12938 (6224 to 26894$)$ & 8334 (2926 to 23738 ) & 10812 (5605 to 20859 ) \\
\hline No asthma & 1772 (1292 to 2431$)$ & 5262 (3541 to 7820 ) & 14167 (10764 to 18645$)$ & 18648 (12 287 to 28301$)$ & 26358 (19658 to 35341$)$ \\
\hline \multicolumn{6}{|l|}{ P6 } \\
\hline Asthma & $353(260$ to 480$)$ & 423 (257 to 697$)$ & 2499 (1068 to 5847$)$ & 900 (465 to 1741$)$ & 1440 (750 to 2766 ) \\
\hline No asthma & 461 (380 to 559$)$ & $853(659$ to 1105$)$ & 4881 (3776 to 6308 ) & 3060 (2253 to 4156$)$ & 3438 (2640 to 4475$)$ \\
\hline Asthma & 1235 (800 to 1905 ) & 1951 (966 to 3941) & 7983 (3254 to 19584$)$ & 11159 (3678 to 33854$)$ & 28167 (9531 to 83242 ) \\
\hline No asthma & 1397 (1167 to 1673 ) & 3144 (2291 to 4313 ) & 14600 (10449 to 20401$)$ & 20777 (13360 to 32313$)$ & 37611 (24638 to 57413$)$ \\
\hline $\mathrm{p}$ Value & 0.4136 & 0.1648 & 0.1906 & 0.2258 & 0.5736 \\
\hline \multicolumn{6}{|l|}{ PspC } \\
\hline Asthma & 1839 (1104 to 3062 ) & 38192 (14 143 to 103133$)$ & 234369 (93344 to 588457 ) & 321962 (153239 to 676456$)$ & 43257 (212726 to 878745$)$ \\
\hline
\end{tabular}

The value is the geometric mean of the $\operatorname{lgG} 1$ antibody titre $(\mathrm{ng} / \mathrm{ml})$ with the $\mathrm{Cl}$ of antibody binding in parentheses.

Comparisons between SPT-positive and SPT-negative groups by the Mann-Whitney test are shown.

$p$ Values $<0.0125$ are considered significant after correcting for multiple testing.

responses of the boys and girls to the other antigens were very similar.

The prevalence of IgG4 antibody to P4 and P6 increased to year 4 but then fell-markedly for P4, and the decrease for P6 was only statistically significant for the non-atopic subjects. The prevalence of IgG4 antibody to the $S$ pneumoniae antigens did not show this fall and increased progressively from year 1 to 5 .

Neither the prevalence of IgG4 antibodies nor the titres were associated with the development of asthma. The titres of IgG1 and IgG4 to P6 and PspA in fact had a consistently positive correlation $(r=0.218, p<0.008$ and $r=0.491, p<0.001$ at year 5 for P6 and PspA, respectively; see table 3 in online supplement). The titres to PspC were significantly correlated at some time points.

Overall, the titres of IgG4 to all of the antigens correlated strongly with each other, including comparisons of the $H$ influenzae and $S$ pneumoniae antigens $(p<0.005$ to $p<0.001$; see table 4 in online supplement).

\section{IgE antibody}

IgE antibody assays required low dilutions of serum so they were performed for the year 5 group and measurements were only made with P6. The IgE was detected in $64 \%$ of serum samples and there was no difference in titre or the prevalence of responses in the SPT-positive and SPT-negative groups.
A number of the higher titres were $>10 \mathrm{ng} / \mathrm{ml}$. When analysed for asthma, non-asthmatic children had significantly higher antiP6 IgE titres $(p<0.05$; figure $3 A)$. In contrast, the children with asthma had significantly higher IgE titres to the major HDM allergens $(p<0.001)$. No correlation was found between the antiHDM and anti-P6 IgE titres $(r=-0.08, p=0.325)$ for the whole cohort but, importantly, there was an inverse relationship between HDM and bacterial IgE titres for the SPT-positive group $(\mathrm{r}=-0.342, \mathrm{p}<0.05)$.

The anti-P6 IgE and IgG1 antibody responses were strongly correlated (figure $3 \mathrm{~B}$ ), but there was no association between the $\mathrm{IgE}$ and IgG4 response.

\section{Immunoglobulin measurements}

Total IgE was also measured at age 5 and there was a negative correlation between the $\operatorname{IgE}$ immunoglobulin and anti-P6 $\operatorname{IgE}$ titre for the SPT-positive group $(\mathrm{r}=-0.329, \mathrm{p}<0.05)$ and a positive trend was observed for the SPT-negative group ( $r=0.408$, $\mathrm{p}=0.09$ ). The expected strong correlation between the anti-Der $\mathrm{p}$ 1 and Der $p 2 \operatorname{IgE}$ titre and total $\operatorname{IgE}(r=0.644, p<0.001)$ was found for SPT-positive children.

IgG1 immunoglobulin was measured at age 3 to compare with the early antibody results. Plasma was unavailable at ages 1 and 2. A weak positive correlation between the anti-P4 IgG1 and total IgG1 immunoglobulin was found $(r=0.259, p<0.005)$, but
Figure 3 (A) Anti-P6 IgE antibody production in asthmatic and nonasthmatic 5-year-old children. The number of children with undetectable $\lg \mathrm{E}$ antibody is shown in parenthesis. (B) Correlation of $\lg \mathrm{G} 1$ and $\lg \mathrm{E}$ antibody binding (ng/ml) to $\mathrm{P} 6$.
A

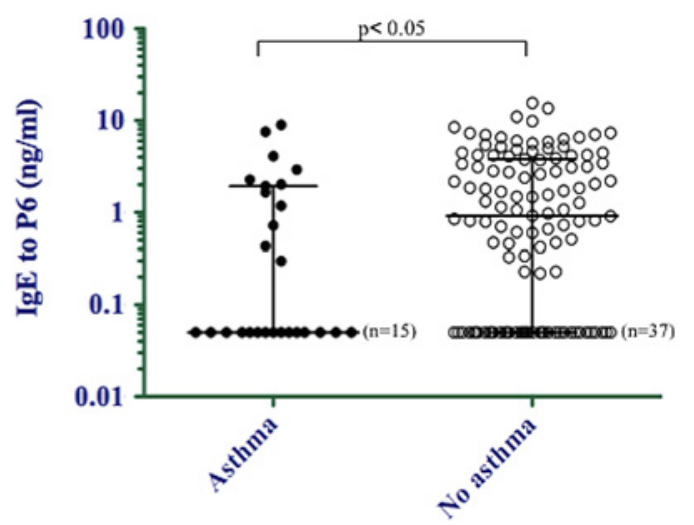

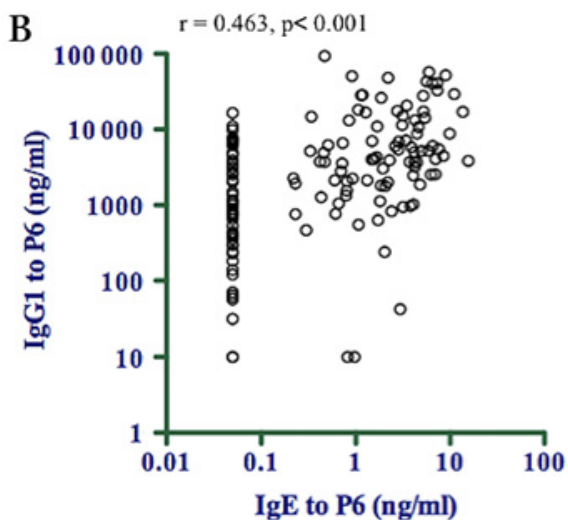


no correlation was found for the other antigens. The IgG1 immunoglobulin levels were similar between the SPT-positive and SPT-negative groups.

\section{DISCUSSION}

The IgG1 results confirm the earlier observation with a subset of this cohort showing that there is an early deficiency in the antibody responses of atopic children to the P6 antigen of $H$ influenzae. The results are now extended to show deficiencies in response to the P4 antigen of $H$ influenzae and the PspA and PspC antigens of $S$ pneumoniae. The most significant differences between the HDM-sensitised and non- sensitised groups were found at either 2 or 3 years, when a rapid increase from infant levels occurred. The decreased titres to the $H$ influenzae antigens and the PspC antigen of $S$ pneumoniae were associated with the development of asthma. This corroborates the finding with an emergency department cohort showing that children with frequent and persistent exacerbations of asthma have low antiP6 IgG1 titres. ${ }^{5}$ The lower IgG1 titres to P4, P6 and PspC were found at a time that precedes the development of anti-allergen $\operatorname{IgE}$ and before asthma was apparent for many children, so they were not the result of bystander effects of prolonged allergic responses. Since IgG switching in humans can be mediated by varying combinations of cytokines produced by Th1, Th2, Th17 and regulatory cells, the deficiencies cannot be easily attributed to a particular $\mathrm{T}$ cell lineage. ${ }^{18}$ They were not generally associated with reduced immunoglobulin levels, indicating that the defects were only found in certain types of antibody responses.

It was previously shown that about $30 \%$ of HDM-sensitised adults and children of school age have IgG4 antibody to the P6 antigen, a response rarely found in non-sensitised subjects. ${ }^{4}$ The results here show that the IgG4 titres in the preschool age groups were low, with no significant association with sensitisation or asthma for any of the antigens. There was also no association of asthma severity with IgG4 titres noted in emergency department admissions. ${ }^{5}$ It appears that the persistence of the IgG4 antibodies in HDM-sensitised subjects only becomes apparent at an older age and is not associated with disease. The positive association of IgG4 and IgG1 titres is also consistent with this. The production of IgG4 is absolutely dependent on Th2 cell help, so it is a marker for Th2 responses. It is interesting that, for the $H$ influenzae antigens, the prevalence of IgG4 fell from 4 to 5 years; this was marked for P4 and was only found in non-sensitised children for P6. Taken together with the results from older subjects, ${ }^{4}$ it seems that IgG4 is downregulated in nearly all non-sensitised subjects but persists in some atopic individuals. It is, however, the low IgG1 and not persistent IgG4 that associates with disease. The bias to the male sex found previously for IgG4 antibody to P6 was also evident, but there was no suggestion that it occurred for the other antigens, even P4. It has been shown that P6 (but not P4) stimulates macrophages by a TLR2-dependent mechanism, ${ }^{19}$ so there may be a sex difference in some aspect of innate immunity.

The detection of IgE antibody to bacteria has been described many times. The results here provide estimates of absolute quantitation of titres to defined antigens. The responses could reach titres typical of the IgE responses of many people to major allergens. This infers that the IgE may be significant in the pathophysiology, and also that the immunological events that underpin the induction of antibacterial IgE, such as Th2 cytokine production, could be as substantial as those induced by allergen. The responses, however, suggest that $\operatorname{IgE}$ is associated with protection from asthma. They corroborate those from an independent large unselected birth cohort where $\operatorname{IgE}$ antibody titres to both $H$ influenzae and $S$ pneumoniae antigens were inversely associated with the risk of asthma, ${ }^{13}$ and subjects with the highest titres of antibacterial IgE had the lowest risk of asthma. Although not initially appreciated, the boosting of $\operatorname{IgE}$ during convalescence from asthma attacks ${ }^{5}$ might be part of a protective response since the antibacterial IgE titres at admission were in fact low. The current study here independently confirms the negative association and also shows that it is evident when asthma first becomes prevalent in children. The protective association might seem paradoxical, but there are at least two general potential pathways. The first is that Th2 cytokines $^{20}$ and Th2 cytokine-induced chemokines ${ }^{21}$ can have powerful anti-inflammatory effects. The sustained Th2 response to colonising bacteria could downregulate Th1 and Th17 responses which could also have a role in the production of disease. $^{22}$ The second is that the IgE antibody itself can enhance antigen capture via Fce receptors on antigen-presenting cells and increase the recruitment of antibacterial $T$ cells which, on balance, have been shown to be polarised to $\mathrm{Th} 1,{ }^{23}$ and these could alter the education of dendritic cells and hence signals to allergen-specific $\mathrm{T}$ cells. ${ }^{24}$ The inverse association of the $\mathrm{IgE}$ to Der p 1 and Der p 2 with the antibacterial IgE points to a downregulation of the anti-allergen $\mathrm{Th} 2$ response. The $\mathrm{IgE}$ antibodies to both $S$ pneumoniae and $H$ influenzae are also highly correlated with each other, ${ }^{13}$ so there seems to be a group of people with a propensity to make IgE antibody to bacterial antigens and they have a reduced risk of asthma. The recent finding that exposure to a diverse range of microbes is inversely related to the risk of asthma points to the possibility that the protective association of antimicrobial IgE antibody and asthma could extend to other organisms. ${ }^{25}$

The point prevalence rates for the detection of colonisation with $H$ influenzae and $S$ pneumoniae are conservatively about $30 \%$ for each organism, ${ }^{26} 27$ with the colonisation episodes typically lasting about 5 weeks. Since the antigens examined have conserved sequences and are found in all isolates, repeated stimulation of the immune response would be expected from the successive waves of colonisation with these organisms every year. Immune responses to $\mathrm{P} 4,{ }^{28} \mathrm{P}^{29}$ and to $\mathrm{PspA}$ and $\mathrm{PspC}^{30}$ are associated with protective immunity, so responses against them could have a bearing on colonisation. The reported increased colonisation of $H$ influenzae in subjects with asthma ${ }^{13}$ would fit the defective IgG1 response reported here.

The low IgG antibody response described here could enhance atopy and asthma by increasing the susceptibility to bacterial infection, and hence tissue damage, by exposure to pharmacologically active bacterial products. ${ }^{6}$ It is also possible that underlying immune responses to the bacteria and allergens influence immune responses to each other when they are copresented at the mucosa to increase the degree of sensitisation. This has been shown, at least for allergens, to be able to increase allergic sensitisation. ${ }^{31}$

It is also possible that the altered antibody responses are just markers that show people with atopy and asthma have alterations in an aspect of their mucosal immune system that extends beyond the response to allergens. These could also extend to virus infections, particularly rhinoviruses which have been implicated as the most frequent virus facilitating both the development and exacerbation of asthma. Recent data have, however, shown that increased bacterial colonisation, independent of viral infection and including both $H$ influenzae and $S$ pneumoniae, has been associated with susceptibility to asthma ${ }^{9}$ and wheezing attacks, ${ }^{12}$ and that young children with asthma 
have increased carriage of pneumococcus. ${ }^{32}$ This is in addition to studies showing that subjects with asthma have an increased susceptibility to invasive bacterial infection, including pneumococcus. ${ }^{33} 34$ Bacterial colonisation was not measured in the current cohort but, in keeping with many reports, virus infection is associated with the development of asthma and is especially associated with atopy. ${ }^{12} 15$

The results here are from a high-risk cohort in which at least one parent had an allergy-associated disease. This might limit the interpretation of the results, but it extends previous observations in an emergency department cohort in which reduced IgG antibody was found in children with frequent and persistent asthma exacerbations..$^{5}$ The association of $\operatorname{IgE}$ antibody with $H$ influenzae and $S$ pneumoniae was previously ascertained in a large unselected birth cohort. ${ }^{13}$

This study has focused on establishing the association between altered immune responses to colonising bacteria and the development of atopy and asthma. It has done this by extending previous observations with the P6 antigens of $H$ influenzae to another protective antigen $\mathrm{P} 4$ and to responses to $S$ pneumoniae, and by verifying the associations with independent cohorts. ${ }^{5} 13$ The development of vaccines against $S$ pneumoniae and non-typable $H$ influenzae is an area of active research. The $S$ pneumoniae polysaccharide- $H$ influenzae protein $\mathrm{D}$ conjugate vaccine has reduced colonisation with both these organisms, ${ }^{35}$ so it might also be used to study how they affect allergy and asthma.

Funding This work was supported by the National Health and Medical Research Council of Australia.

\section{Competing interests None.}

Ethics approval Ethics approval was provided by the Princess Margaret Hospital human ethics committee.

Contributors BJH and WRT were responsible for the conception and design of the study and for writing the manuscript. BJH performed the majority of the data analysis, aided by LYC, CEE and LJP. GZ provided expert statistical advice and assisted with the data analysis. BJH, TKH, W-AS and WRT were responsible for the design and validation of the conserved protein antigens used in the study. MMHK, PDS and PGH assisted in interpretation of the analysis and were involved in advice and feedback of the manuscript. All authors gave final approval of the version to be published.

Provenance and peer review Not commissioned; externally peer reviewed.

\section{REFERENCES}

1. Simpson A, Soderstrom L, Ahlstedt S, et al. IgE antibody quantification and the probability of wheeze in preschool children. J Allergy Clin Immunol 2005;116:744-9.

2. Papadopoulos NG, Stanciu LA, Papi A, et al. A defective type 1 response to rhinovirus in atopic asthma. Thorax 2002;57:328-32.

3. Message SD, Laza-Stanca V, Mallia $P$, et al. Rhinovirus-induced lower respiratory illness is increased in asthma and related to virus load and Th1/2 cytokine and IL-10 production. Proc Natl Acad Sci U S A 2008;105:13562-7.

4. Hales BJ, Pearce LJ, Kusel MM, et al. Differences in the antibody response to a mucosal bacterial antigen between allergic and non-allergic subjects. Thorax 2008;63:221-7

5. Hales BJ, Martin AC, Pearce LJ, et al. Anti-bacterial lgE in the antibody responses of house dust mite allergic children convalescent from asthma exacerbation. Clin Exp Allergy 2009;39:1170-8.

6. Woodfolk JA. Microbes and man: an evolving story in asthma. Clin Exp Allergy 2009;39:1112-14.

7. Busse WW, Lemanske RF Jr, Gern JE. Role of viral respiratory infections in asthma and asthma exacerbations. Lancet 2010;376:826-34.

8. Olenec JP, Kim WK, Lee WM, et al. Weekly monitoring of children with asthma for infections and illness during common cold seasons. J Allergy Clin Immunol 2010:125:1001-6.e1.

9. Bisgaard $\mathbf{H}$, Hermansen MN, Buchvald $\mathbf{F}$, et al. Childhood asthma after bacterial colonization of the airway in neonates. N Engl J Med 2007:357:1487-95.
10. Hilty M, Burke $\mathrm{C}$, Pedro $\mathrm{H}$, et al. Disordered microbial communities in asthmatic airways. PLoS One 2010;5:e8578.

11. Jackson DJ, Gangnon RE, Evans MD, et al. Wheezing rhinovirus illnesses in early life predict asthma development in high-risk children. Am J Respir Crit Care Med 2008;178:667-72.

12. Bisgaard $\mathbf{H}$, Hermansen MN, Bonnelykke $\mathrm{K}$, et al. Association of bacteria and viruses with wheezy episodes in young children: prospective birth cohort study. $B M$ 2010;341:c4978.

13. Hollams EM, Hales BJ, Bachert $\mathrm{C}$, et al. Th2-associated immunity to bacteria in asthma in teenagers and susceptibility to asthma. Eur Respir J 2010;36: 509-16

14. Kusel MM, de Klerk NH, Holt PG, et al. Role of respiratory viruses in acute upper and lower respiratory tract illness in the first year of life: a birth cohort study. Pediatr Infect Dis J 2006;25:680-6.

15. Kusel MM, de Klerk NH, Kebadze T, et al. Early-life respiratory viral infections, atopic sensitisation, and risk of subsequent development of persistent asthma. J Allergy Clin Immunol 2007:119:1105-10.

16. Schuurman J, Lourens TE, Perdok GJ, et al. Mouse/human chimeric lgE antibodies directed to the house dust mite allergen Der p 2. Int Arch Allergy Immunol 1995:107:465-6.

17. Schuurman J, Perdok GJ, Mueller GA, et al. Mouse/human chimeric IgG1 and lgG4 antibodies directed to the house dust mite allergen Der $\mathrm{p} 2$ : use in quantification of allergen specific IgG. Clin Exp Allergy 1997;27:1095-102.

18. Avery DT, Bryant VL, Ma CS, et al. IL-21-induced isotype switching to IgG and IgA by human naive B cells is differentially regulated by IL-4. J Immunol 2008;181:1767-79.

19. Punturieri A, Copper P, Polak T, et al. Conserved nontypeable Haemophilus influenzae-derived TLR2-binding lipopeptides synergize with IFN-beta to increase cytokine production by resident murine and human alveolar macrophages. J Immunol 2006;177:673-80.

20. Hart PH, Vitti GF, Burgess DR, et al. Potential antiinflammatory effects of interleukin 4: suppression of human monocyte tumor necrosis factor alpha, interleukin 1, and prostaglandin E2. Proc Natl Acad Sci U S A 1989;86:3803-7.

21. Taylor BC, Zaph C, Troy AE, et al. TSLP regulates intestinal immunity and inflammation in mouse models of helminth infection and colitis. J Exp Med 2009;206:655-67.

22. Durrant DM, Metzger DW. Emerging roles of $\mathrm{T}$ helper subsets in the pathogenesis of asthma. Immunol Invest 2010;39:526-49.

23. Epton MJ, Hales BJ, Thompson PJ, et al. T cell cytokine responses to outer membrane proteins of Haemophilus influenzae and the house dust mite allergens Der p 1 in allergic and non-allergic subjects. Clin Exp Allergy 2002;32:1589-95.

24. Larsson $\mathbf{K}$, Lindstedt $\mathrm{M}$, Lundberg $\mathrm{K}$, et al. CD4+ T cells have a key instructive role in educating dendritic cells in allergy. Int Arch Allergy Immunol 2009;149:1-15.

25. Ege MJ, Mayer M, Normand AC, et al; GABRIELA Transregio 22 Study Group. Exposure to environmental microorganisms and childhood asthma. N Engl J Med 2011;364:701-9

26. Hendley J0, Hayden FG, Winther B. Weekly point prevalence of Streptococcus pneumoniae, Hemophilus influenzae and Moraxella catarrhalis in the upper airways of normal young children: effect of respiratory illness and season. APMIS 2005:113:213-20.

27. Garcia-Rodriguez JA, Fresnadillo Martinez MJ. Dynamics of nasopharyngeal colonization by potential respiratory pathogens. J Antimicrob Chemother 2002;50 (Suppl S2):59-73.

28. Hotomi M, Ikeda $Y$, Suzumoto $M$, et al. A recombinant P4 protein of Haemophilus influenzae induces specific immune responses biologically active against nasopharyngeal colonization in mice after intranasal immunization. Vaccine 2005;23:1294-300

29. Abe N, Kodama S, Hirano T, et al. Nasal vaccination with $\mathrm{CpG}$ oligodeoxynucleotide induces protective immunity against non-typeable Haemophilus influenzae in the nasopharynx. Laryngoscope 2006;116:407-12

30. Daniels CC, Coan $\mathrm{P}$, King J, et al. The proline-rich region of pneumococcal surface proteins $A$ and $C$ contains surface-accessible epitopes common to all pneumococc and elicits antibody-mediated protection against sepsis. Infect Immun 2010;78:2163-72.

31. Blumchen K, Gerhold K, Schwede M, et al. Effects of established allergen sensitisation on immune and airway responses after secondary allergen sensitisation. J Allergy Clin Immunol 2006;118:615-21.

32. Jounio $\mathbf{U}$, Juvonen $\mathrm{R}$, Bloigu $A$, et al. Pneumococcal carriage is more common in asthmatic than in non-asthmatic young men. Clin Respir J 2010:4:222-9.

33. Talbot TR, Hartert TV, Mitchel E, et al. Asthma as a risk factor for invasive pneumococcal disease. N Engl J Med 2005;352:2082-90.

34. Juhn YJ, Kita H, Yawn BP, et al. Increased risk of serious pneumococcal disease in patients with asthma. J Allergy Clin Immunol 2008;122:719-23.

35. Prymula R, Kriz P, Kaliskova E, et al. Effect of vaccination with pneumococcal capsular polysaccharides conjugated to Haemophilus influenzae-derived protein D on nasopharyngeal carriage of Streptococcus pneumoniae and $\mathrm{H}$ influenzae in children under 2 years of age. Vaccine 2009:28:71-8. 